Supplement of

\title{
Quantifying the sensitivity of aerosol optical properties to the parameterizations of physico-chemical processes during the 2010 Russian wildfires and heatwave
}

\section{Laura Palacios-Peña et al.}

Correspondence to: Pedro Jiménez-Guerrero (pedro.jimenezguerrero@um.es)

The copyright of individual parts of the supplement might differ from the CC BY 4.0 License. 


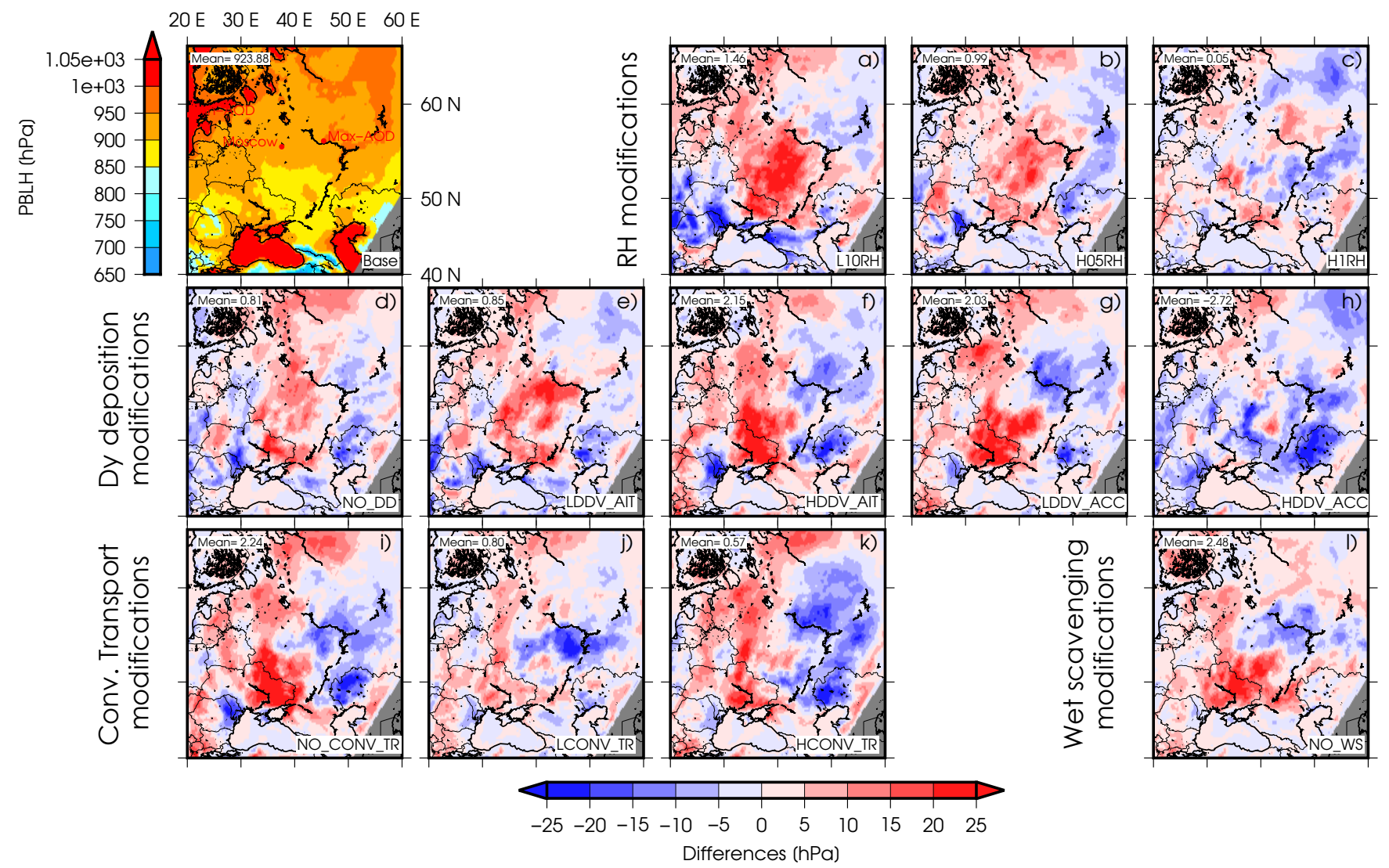

Figure S1. Mean PBL height during the target episode and differences between all the experiments and the base case. 

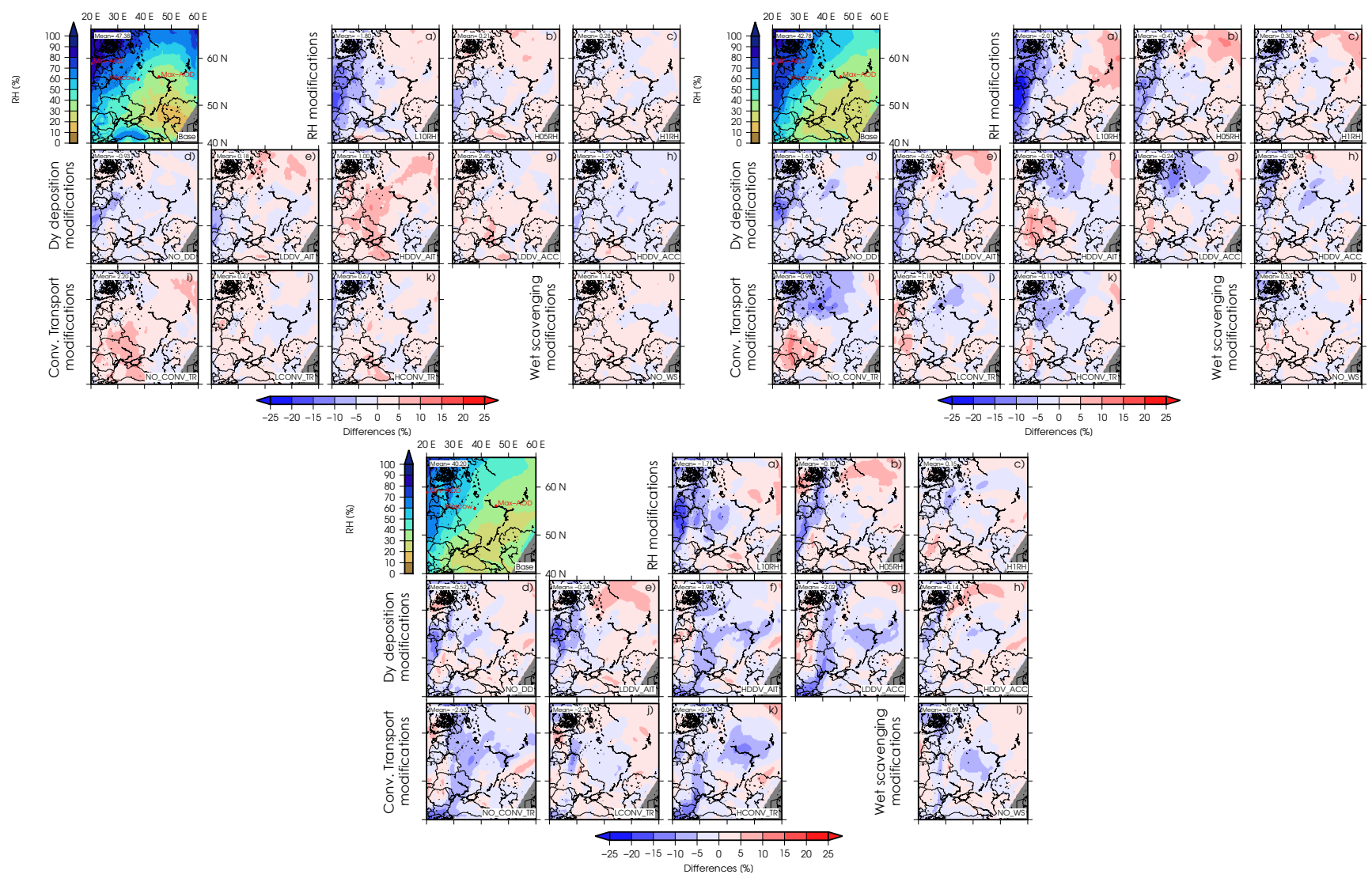

Figure S2. Temporal mean RH values at 1000 (top-left), 850 (top-right) and $750 \mathrm{hPa}$ (bottom) levels during the target episode and differences between all the experiments and the base case. 



Figure S3. Temporal mean of low cloud fraction (below $680 \mathrm{hPa}$, top-left), medium (between 680 and $440 \mathrm{hPa}$, top-right) and high (above $440 \mathrm{hPa}$, bottom) during the target episode and differences between all the experiments and the base case. 
MAX-AOD
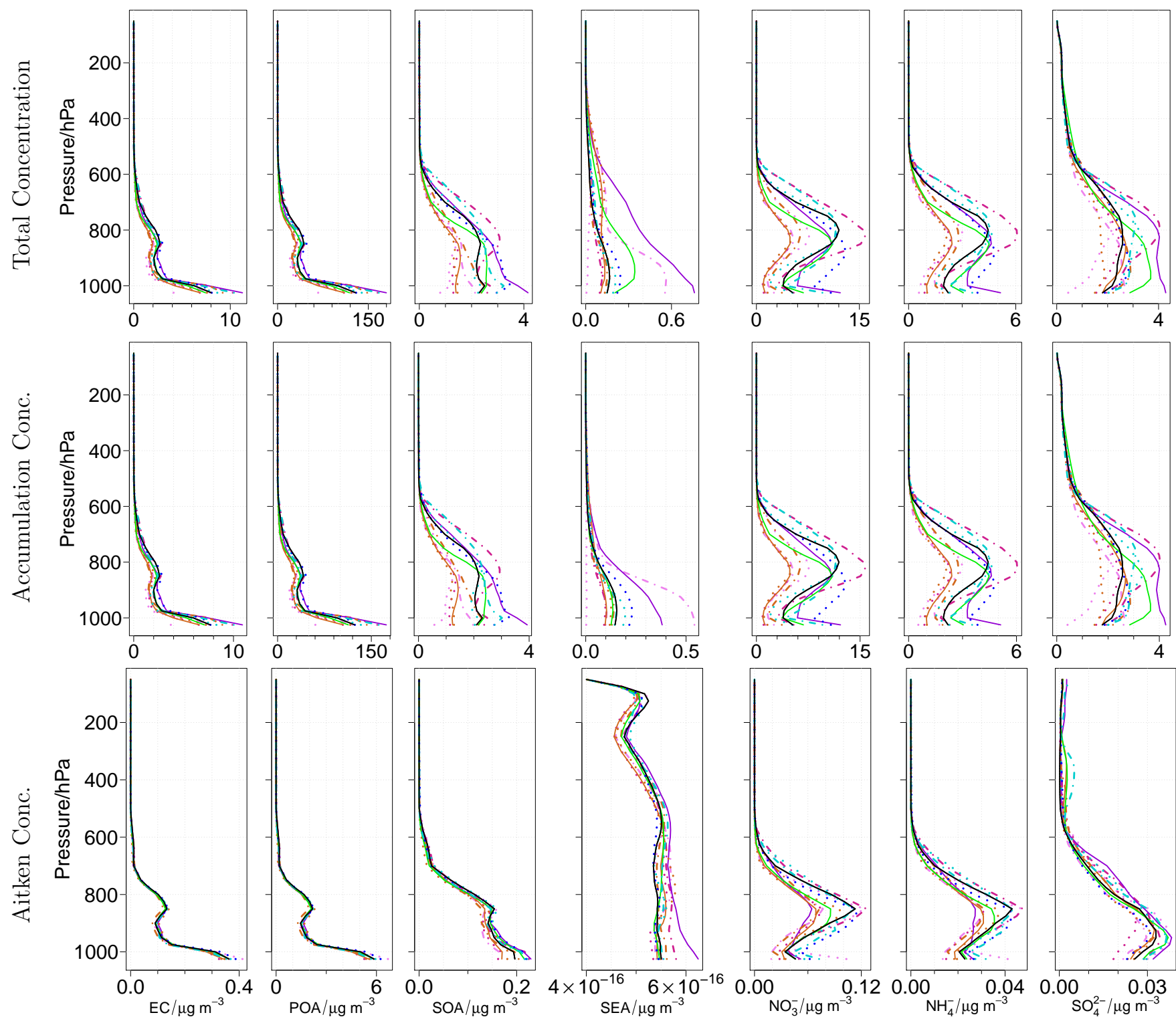

Figure S4. Total concentration profiles over the MAX-AOD location. From second to bottom rows, columns display concentration of EC, $\mathrm{POA}, \mathrm{SOA}, \mathrm{SEA}, \mathrm{NO}_{3}^{-}, \mathrm{NH}_{4}^{-} \& \mathrm{SO}_{4}^{2-}$. First row is for total concentration, second in the accumulation mode and bottom in Aitken. 

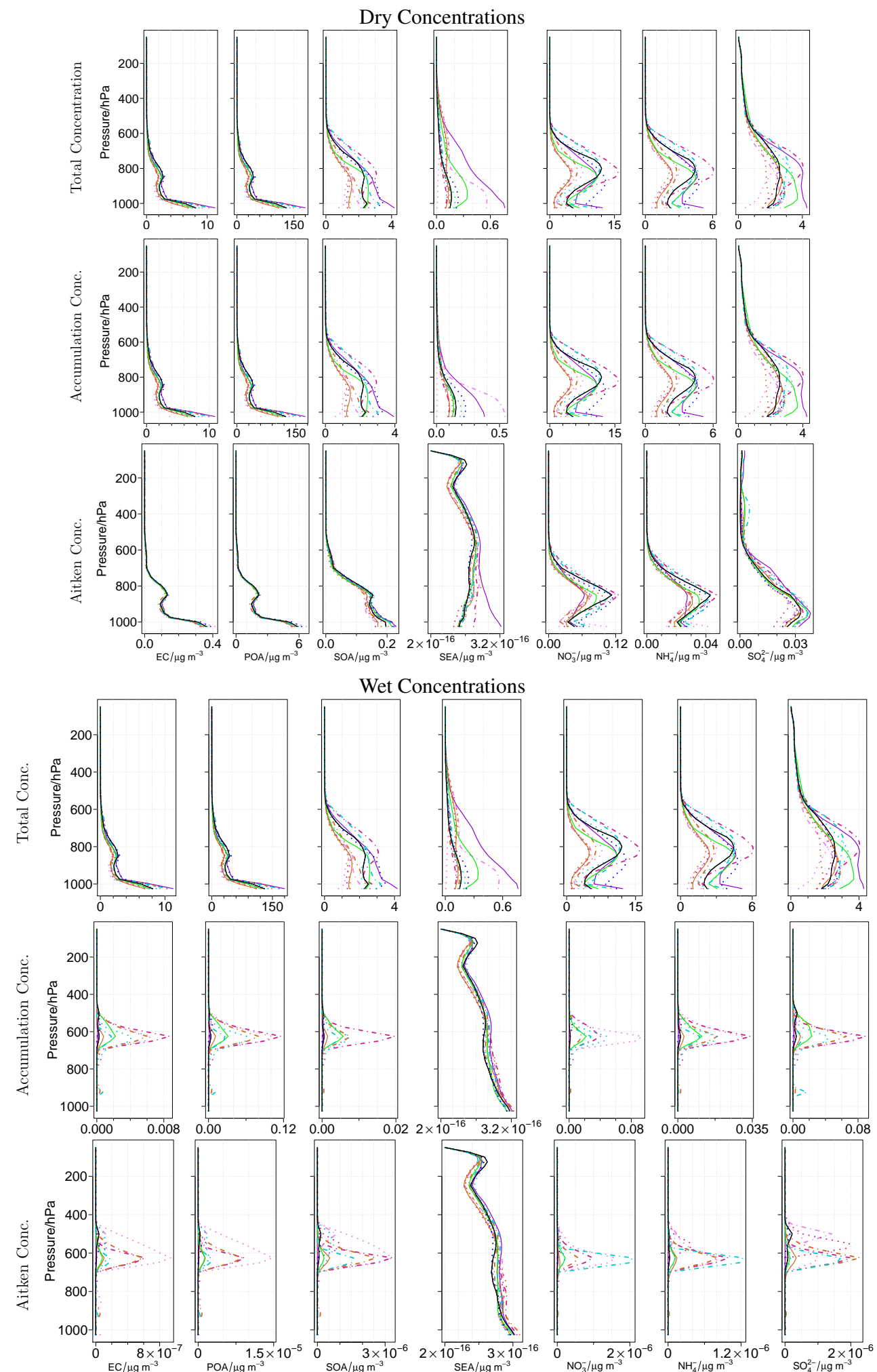

Figure S5. As Figure 0.4 but for wet (top) and dry (bottom) concentrations. 
MIN-AOD
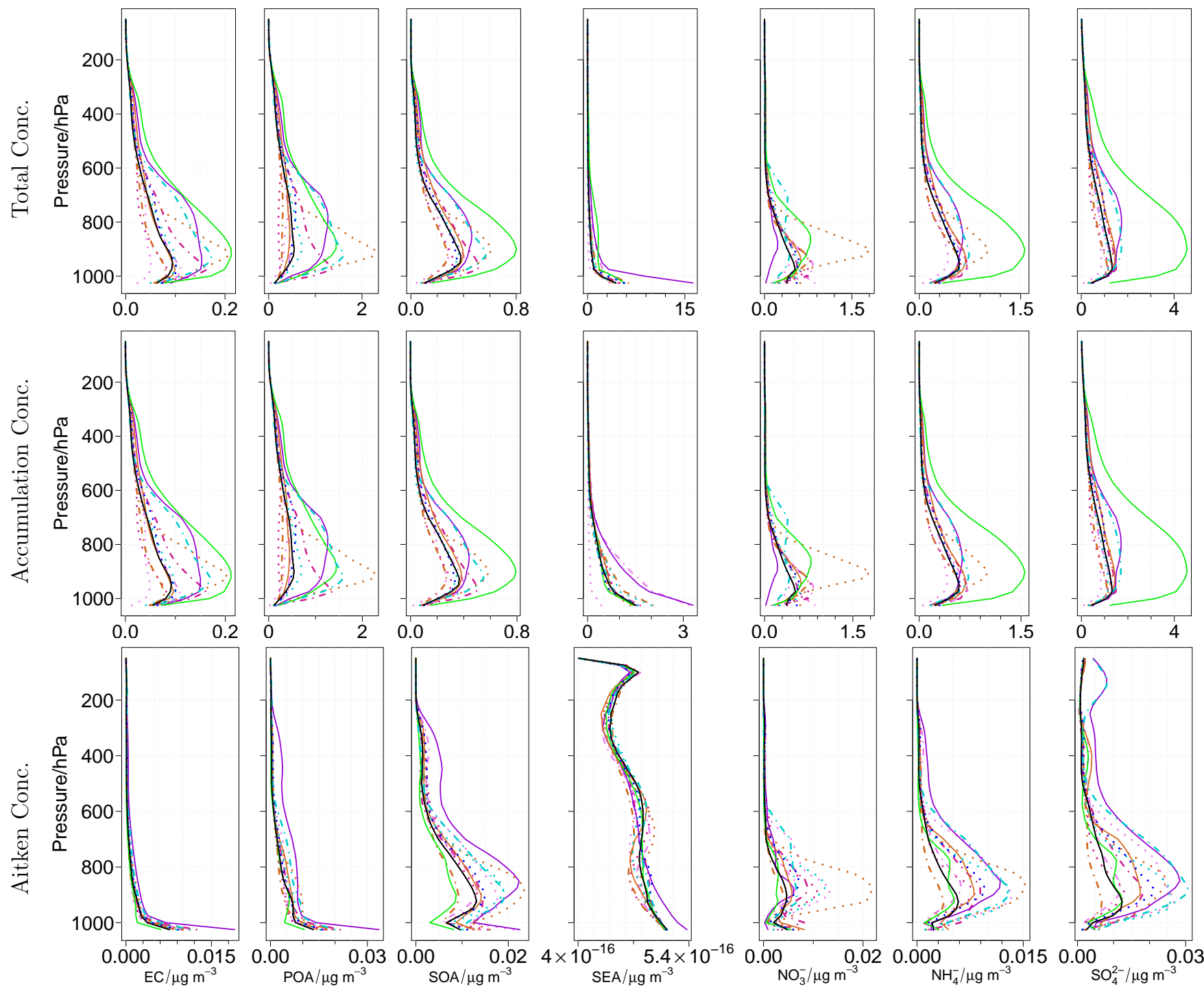

Figure S6. As Figure 0.4 but over the Min-AOD location. 

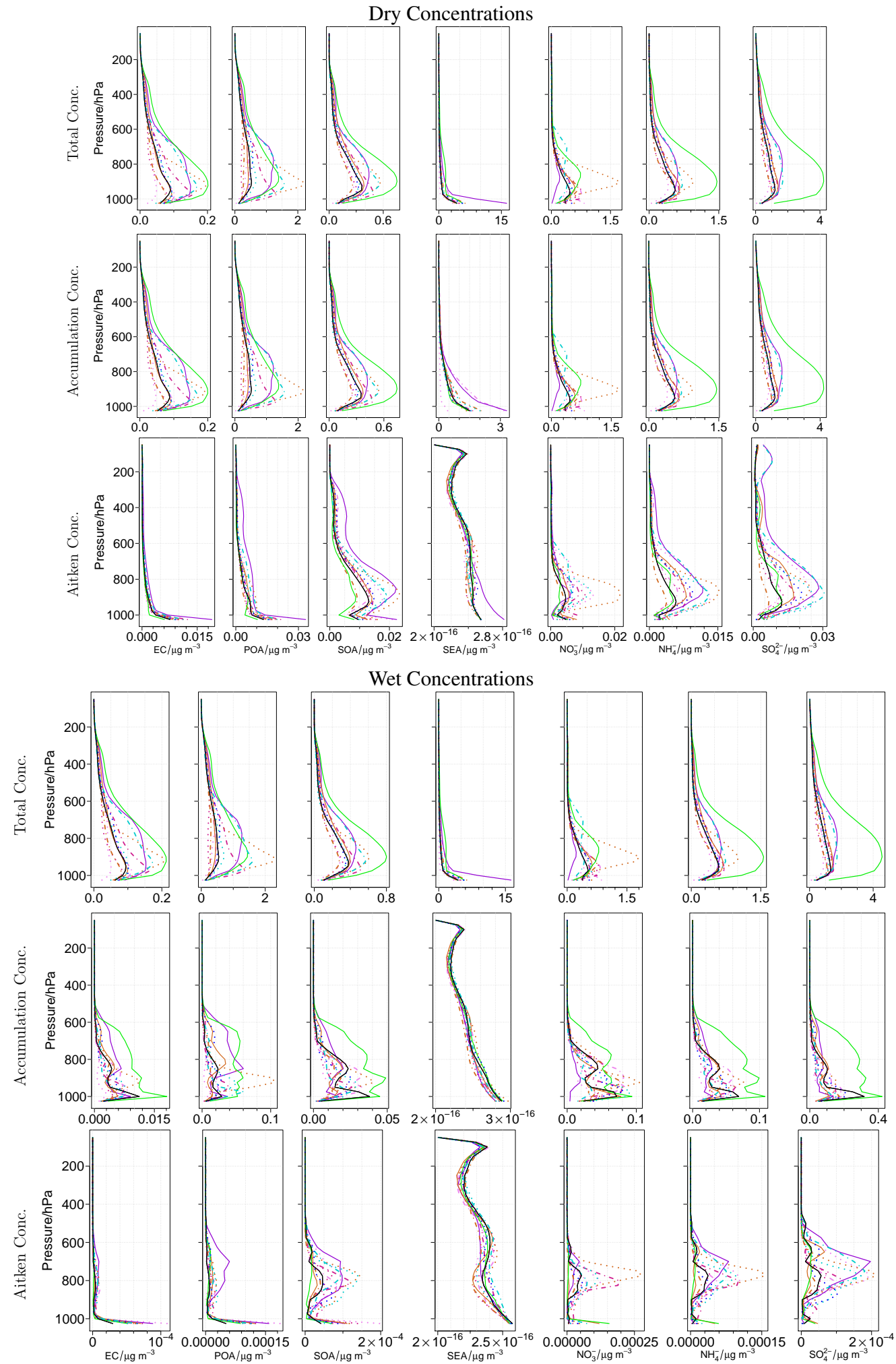

Figure S7. As Figure 0.6 but for wet (top) and dry (bottom) concentrations. 
MOSCOW
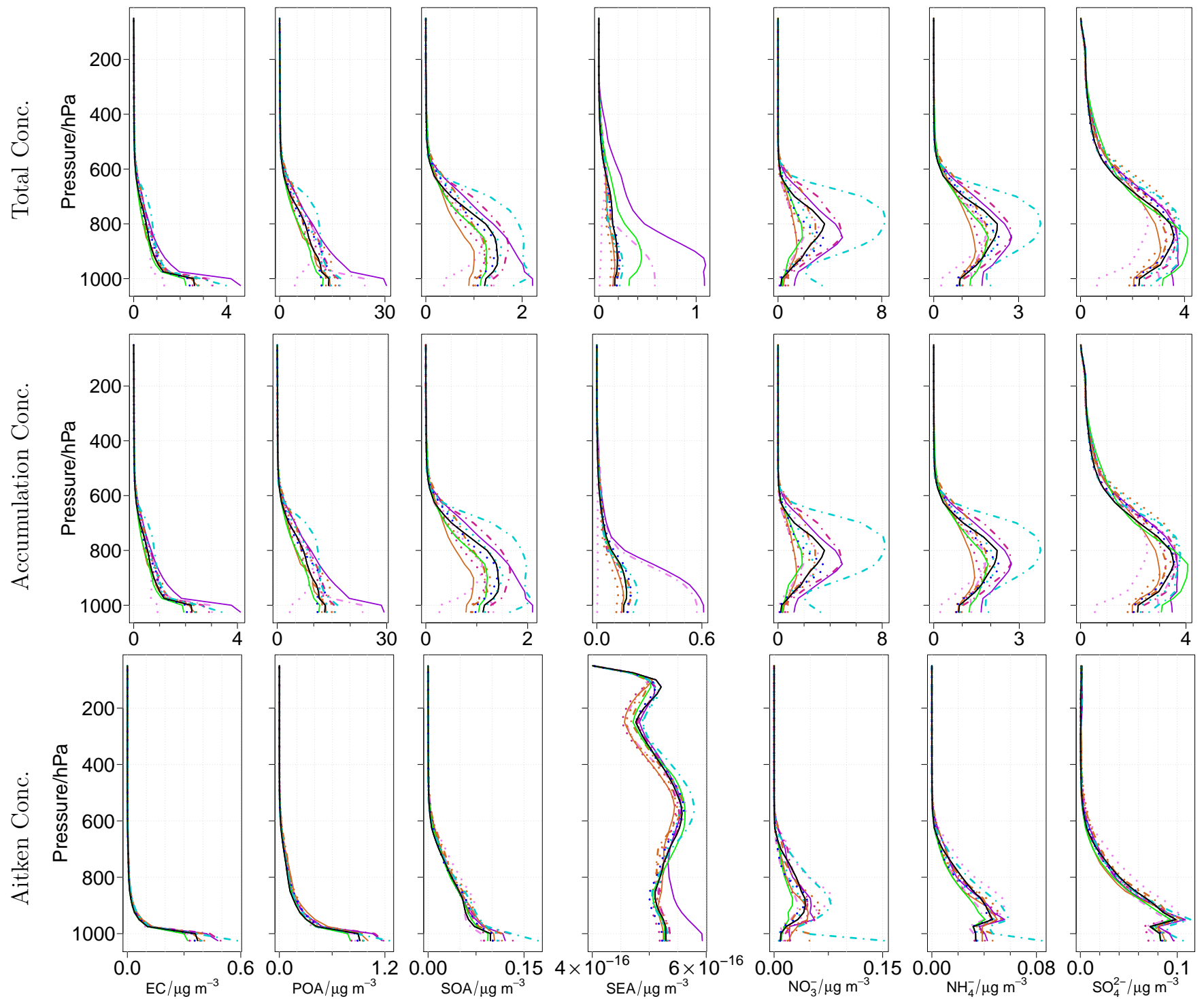

Figure S8. As Figure 0.4 but over Mowcow location. 

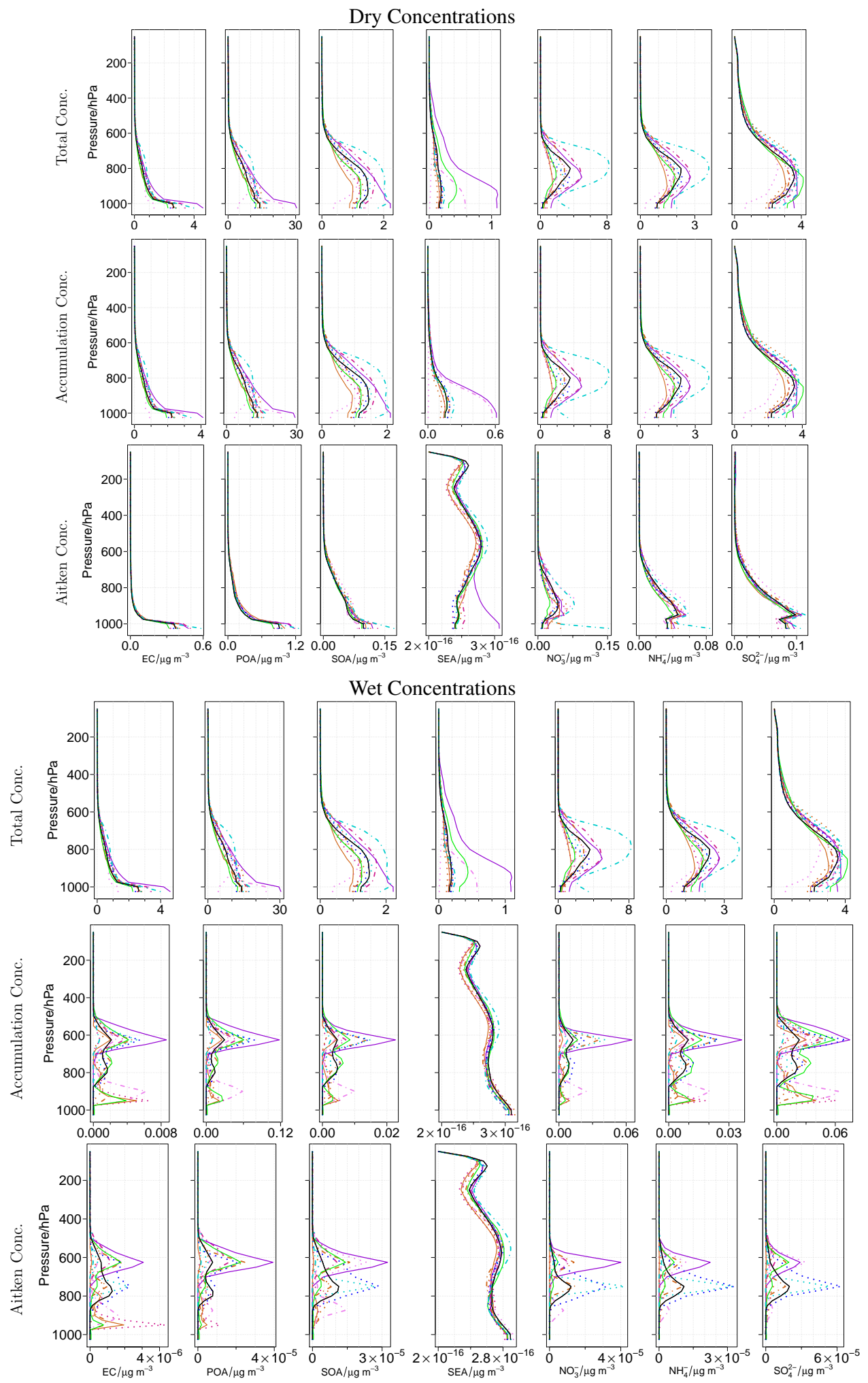

Figure S9. As Figure 0.8 but for wet (top) and dry (bottom) concentrations. 